\title{
Desigualdad de género en procesos educativos incidente en violencia hacia la mujer
}

\section{Gender inequality in educational processes that incide on violence against women}

Gloria Solís-Beltrán ${ }^{1}$, Mario Fernández-Ronquillo ${ }^{2}$, Luis Solís-Granda ${ }^{3}$, Carlos Terán-Puente ${ }^{4}$

INFORMACIÓN DEL ARTÍCULO

Fecha de recepción: 25 de Octubre de 2017

Fecha de aceptación: 14 de Marzo de 2018

1 Magíster en Gerencia Educativa, Universidad Estatal de Milagro. Docente en la Universidad Estatal de Milagro-Ecuador.

E-mail: gsolisb@unemi.edu.ec

Magíster en Dirección y Administración de Empresas, Universidad Tecnológica Empresarial de Guayaquil. Docente en la Universidad Estatal de Milagro-Ecuador.

E-mail:mfernandezr@unemi.edu.ec

${ }^{3}$ Magíster en Dirección y Administración de Empresas, Universidad Tecnológica Empresarial de Guayaquil. Docente en la Universidad Estatal de Milagro-Ecuador. E-mail: 1solisg@unemi.edu.ec

4 Master of Primary Health Care Management, Istituto Superiore di Sanità. Docente en la Universidad Estatal de Milagro-Ecuador.

E-mail: cteranp@unemi.edu.ec

CITACIÓN: Solís-Beltrán, G., Fernández-Ronquillo, M., Solís-Granda, L., \& Terán-Puente, C. (2018). Desigualdad de género en procesos educativos incidente en violencia hacia la mujer. Podium, 33, 13-24.

doi:10.31095/podium.2018.33.2

ENLACE DOI:

http://dx.doi.org/10.31095/podium.201 8.33 .2

\section{Resumen}

La igualdad de género es considerada un asunto de derecho y condición para la salud y el desarrollo de las familias y las sociedades; muchos autores consideran que la educación es clave para reducir las desigualdades de grupo. El objetivo de esta investigación es indagar sobre el impacto de la desigualdad de género en los procesos educativos y su incidencia en la violencia hacia la mujer en Ecuador. La revisión de literatura, el debate y discusión sobre el tema se ha ampliado y profundizado en los últimos años; evidenciando, una mayor sensibilización y concientización social. Esto, ha hecho posible visualizar estudios de casos relacionados con la participación de la mujer en espacios educativos, y la violencia de género en general. Aunque el desarrollo de estudios de género en Ecuador no ha sido prolífero, se espera que estableciendo un marco legal y teórico, esta situación cambie en los próximos años.

\section{Palabras Clave:}

Desigualdad de género, procesos educativos, violencia, mujer, equidad de género.

\section{Clasificación JEL: I14 - I24.}

\section{Abstract}

Gender equality is considered a legal matter and a requirement for family and society health and development. Many authors believe that education is key to reducing group inequalities. The aim of this research is to investigate the impact of gender inequality on educational processes and its incidence on violence against women in Ecuador. Literature review, debate and discussion on the subject have expanded and deepened in recent years; greater social awareness has been evidenced. This has made it possible to visualize case studies related to female educational spaces, and to gender violence in general. Although the development of gender studies has not been prolific in Ecuador, it is expected that this situation will change in the coming years because of the establishment of a legal and theoretical framework.

\section{Keywords:}

Gender inequality, educational processes, violence, women, gender equity.

JEL Classification: I14 - I24. 


\section{Introducción}

La igualdad de género y los derechos de las mujeres son fundamentales para abordar los asuntos pendientes de los Objetivos de Desarrollo Sostenible y acelerar el desarrollo mundial más allá de 2015; así, la igualdad de género es considerada un asunto de derecho y condición para la salud y el desarrollo de las familias y las sociedades, un motor del crecimiento económico (Naciones Unidas, 2015); de hecho, existen muchas razones para preocuparse por las desigualdades de género existentes en importantes dimensiones relacionadas con el bienestar, como educación, salud, empleo o salario (Klasen y Lamanna, 2009).

Como uno de los retos del nuevo milenio, las Naciones Unidas (2015), considera que:

Si se facilita a las mujeres y niñas igualdad en el acceso a la educación, atención médica, un trabajo decente y representación en los procesos de adopción de decisiones políticas y económicas, se impulsarán las economías sostenibles y se beneficiará a las sociedades y a la humanidad en su conjunto.

CEPAL (2016) ha establecido como objetivo de desarrollo sostenible el lograr la igualdad entre los géneros y empoderar a todas las mujeres y las niñas, específicamente, se plantea como metas: Poner fin a todas las formas de discriminación contra todas las mujeres y las niñas en todo el mundo, eliminar todas las formas de violencia contra todas las mujeres y las niñas en los ámbitos público y privado, incluidas la trata y la explotación sexual y otros tipos de explotación, así como velar por la participación plena y efectiva de las mujeres y la igualdad de oportunidades de liderazgo a todos los niveles de la adopción de decisiones en la vida política, económica y pública, entre otros.

La igualdad de género ha sido una prioridad clave para la Unión Europea durante las últimas cinco décadas, y sigue siendo así (Freidenvall, 2015); como lo reconoce García (2000), aunque los gobiernos mundiales vienen discutiendo como lograr una situación más equilibrada y justa para la humanidad, es recientemente cuando han comenzado a identificarse en términos concretos las condiciones esenciales que en conjunto se requieren para alcanzar esa meta. Algunos de estos cambios han contribuido a reducir la desigualdad de género o por lo menos a hacerla evidente entre sectores más amplios de la población (Aguiar y Gutiérrez, 2017).

La búsqueda de la igualdad de género en las últimas décadas, ha pasado de los lineamientos generales a la formulación de políticas, en parte, porque se estima que ésta contribuye a aumentar el crecimiento económico; sin embargo, a pesar de la multitud de políticas y estrategias para promover la igualdad de género, persisten significativas desigualdades y las 
estimaciones sugieren que éstas continuarán durante muchas décadas (Perrons, 2017).

Dada la conexión obvia entre el éxito educativo y el mercado laboral, se considera que la educación es clave para reducir las desigualdades de grupo; en particular, se cree que la escolaridad desempeña un papel fundamental en el éxito de los grupos raciales/étnicos, como los asiáticos, y las luchas continuas de otros, por ejemplo: negros, nativos americanos e hispanos (Bobbitt, 2007).

Aunque la participación de las mujeres en altos cargos directivos y gerenciales se ha incrementado en las últimas décadas, su participación en la cima de la escala organizacional sigue siendo muy reducida; las barreras para alcanzar tales posiciones van más allá de la formación, pues hoy en día hay paridad a nivel educativo, incluso las mujeres en diferentes ámbitos han superado al varón, el problema está más centrado en los estereotipos vinculados al género, los cuales están en clara disonancia con el rol de liderazgo, que suele estar compuesto por características masculinas (Contreras, Pedraza y Mejía, 2012).

Esta investigación tiene por objeto indagar sobre el impacto de la desigualdad de género en los procesos educativos y su incidencia en la violencia hacia la mujer en Ecuador. Partiendo de esta sección introductoria, se presenta un abordaje teórico sobre la desigualdad de género; luego, su papel en los procesos educativos, como preámbulo a la discusión de la violencia de género. Así, antes de presentar las reflexiones finales, se revisan los avances en el Ecuador.

\section{La desigualdad de género}

La Igualdad de Género es definida como "la igualdad de derechos, responsabilidades y oportunidades de las mujeres y los hombres, y las niñas y los niños" (UNESCO, 2015). La discriminación de género es el trato perjudicial de un individuo o grupo debido al género; no es biológicamente definida para nadie más que por la sociedad, las normas, la cultura, las personas, etc., que crean el ambiente de discriminación de género; generalmente, la desigualdad de género o la palabra discriminación se utiliza para las "mujeres", porque se consideran la parte más inferior y más débil de la sociedad (Shastri, 2014).

La desigualdad de género toma formas diferentes, dependiendo de la estructura económica y de la organización social de una Sociedad en particular y de la cultura de cualquier grupo particular dentro de esa sociedad; aunque se habla de desigualdad de género, suelen ser las mujeres quienes resultan desfavorecidas con respecto a los hombres (Lorber, 2010).

La violencia contra la mujer es la violación más frecuente y universal de los derechos humanos; no conoce fronteras geográficas, ni límite de edad, ni distinción de clase, ni diferencias culturales o raciales, y tiene fuertes implicaciones para 
la igualdad de género, la inclusión social y la salud (Unión Europea, 2010); está presente en los distintos ámbitos del quehacer social, laboral, educativo, comunitario, de la salud, y familiar (Camacho, 2014).

Aguiar y Gutiérrez (2017) a partir de diferentes estadísticas, analizan tres procesos relacionados con la desigualdad de género en México: transición demográfica, desarrollo humano $\mathrm{y}$ participación femenina en el mercado laboral. Se destaca, entre los cambios, el que los hogares tienen menos integrantes, y ello ha disminuido las cargas de la maternidad y del hogar para la mujer; pero los cambios positivos han sido contrarrestados por situaciones como el embarazo adolescente, el incremento de hogares monoparentales de jefatura femenina y la prevalencia de la poca participación masculina en actividades domésticas. Destacan que, México mejoró en el Índice Desigualdad de Género por el aumento de la presencia femenina en el congreso, y que los ingresos representan la mayor desigualdad.

\section{Desigualdad de género en los procesos educativos}

La desigualdad de género en la sociedad es un gran problema que no puede ser abordado solo por la educación; sin embargo, los sistemas educativos y las escuelas pueden contribuir a la igualdad de género en lugar de sostener las desigualdades (Aikman y Unterhalter, 2007). Un importante foco de la literatura del tema, ha sido examinar el impacto de la desigualdad de género en la educación sobre el crecimiento económico (Klasen y Lamanna, 2009); dada la conexión evidente entre el éxito educativo y los resultados del mercado de trabajo, muchos consideran que la educación es clave para reducir las desigualdades de grupo (Bobbitt, 2007).

Las actitudes hacia la igualdad están evolucionando, pero la generación más joven de hoy no es inmune a los estereotipos y disparidades de género; persisten las desigualdades de género en la educación, en términos de preferencias de los sujetos de estudio, desempeño y patrones de participación (Comisión Europea, 2016); la presencia de las mujeres (hombres) en algunos programas educativos puede facilitarse o restringirse de modo que una consecuencia consista en obstaculizar la incorporación de ellas (ellos) a ciertas opciones laborales creando brechas y diferencias en los salarios de algunos y limitando los niveles de ingresos de las otras (Rodríguez y Limas, 2017).

La desigualdad en las ocupaciones está tomando nuevas formas, en lugar de disminuir y, a pesar de su inversión en educación, las mujeres jóvenes son todavía dos veces más probables que los hombres jóvenes de ser económicamente inactivos (Comisión Europea, 2016). La UNESCO (2012) también reporta que la participación femenina en la educación terciaria disminuye de forma notable en la transición entre la maestría y el doctorado; es aún más significativo el descenso entre quienes se incorporan al trabajo académico y a la investigación (Ordorika, 2015).

La violencia de género relacionada con 
la escuela es un fenómeno inaceptable que debilita los esfuerzos por facilitar educación de buena calidad y alcanzar la Educación para Todos; afecta a la asistencia, el aprendizaje y la finalización de todos los estudiantes, y tiene repercusiones negativas más amplias para las familias y las comunidades (UNESCO, 2015).

Es en las escuelas donde se produce este tipo de violencia de género, pero también es allí donde se le puede poner fin; la escuela debería ser un entorno de aprendizaje donde se cuestionen y transformen las normas sociales y las desigualdades de género, entre ellas las actitudes y las prácticas que condonan la violencia (UNESCO, 2015). Entre las acciones fructíferas se incluyen el cambio curricular, enfrentar el acoso sexual en la escuela y sus alrededores, la formación de maestros sensibles al género y la atención a los diversos estilos de aprendizaje (Aikman y Unterhalter, 2007).

Castillo y Gamboa (2013), consideran que el proceso de socialización que se da en los primeros años escolares contribuye a la formación de la identidad social, específicamente al rol género, y que el papel de la educación es fundamental para visibilizar las desigualdades existentes en la sociedad, muchas de las cuales se han considerado parte de ella. Discuten la vinculación existente entre el papel de la educación y el rol del género a través del abordaje de temas como las relaciones sexistas en la educación, formas de discriminación y transversalización de género en la educación.
Martínez y Bivort (2013) realizan una discusión teórica del valor de los estereotipos como constructos psicológicos para la comprensión y abordaje crítico y transformador de las inequidades de género en educación. Los autores analizan diversos aspectos de la educación, estableciendo un énfasis en el análisis de la educación universitaria y el trabajo académico. Primeramente, discuten la igualdad en educación como un objetivo que tradicionalmente se ha simplificado como equidad, para luego introducir los estereotipos de género como construcciones teóricas crecientemente estudiadas y muy útiles, en tanto se encontrarían cercanas a las bases simbólicas de las brechas de género en educación, afectando tanto las estructuras educacionales, como los niveles más subjetivos de la relación entre género y conocimiento.

Buchmann, DiPrete, y McDaniel (2008) a partir de la revisión de investigaciones empíricas y las perspectivas teóricas sobre las desigualdades de género en el desempeño educativo y el logro desde la primera infancia hasta la edad adulta, plantean que, gran parte de la literatura sobre niños y adolescentes atiende a las diferencias de rendimiento entre niñas y niños; y que, el logro en la escuela primaria y secundaria está vinculado con el nivel de educación que finalmente se logra incluyendo la terminación de la escuela secundaria, la matrícula en la educación postsecundaria, la terminación universitaria y las experiencias académicas de posgrado $\mathrm{y}$ profesional. Con base en el trabajo realizado, recomiendan tres direcciones 
para futuras investigaciones: (A) esfuerzos interdisciplinarios para comprender las diferencias de género en el desarrollo cognitivo y las habilidades no cognitivas en la primera infancia, B) investigación sobre la estructura y las prácticas de la escolarización, y (C) análisis de cómo las diferencias de género podrían amplificar otros tipos de desigualdades, como las desigualdades raciales, étnicas, de clase o de la natividad.

\section{Desigualdad de género y violencia}

La relación entre género y violencia es compleja; las desigualdades de género tienen un amplio impacto en la sociedad, donde a menudo, aumentan el riesgo de actos de violencia por parte de los hombres contra las mujeres (World Health Organization, 2009). La violencia de género está enraizada en las desigualdades estructurales entre hombres y mujeres; es a la vez causa y consecuencia de la desigualdad de género, incorporando una variedad de crímenes y comportamientos incluyendo abuso físico, emocional, sexual, psicológico y económico (Lombard, 2015).

En la Asamblea General de las Naciones Unidas (1993) la violencia contra la mujer fue definida como:

Todo acto de violencia de género que da lugar a, o es probable que resulte en daño o sufrimiento físico, sexual $o$ psicológico a las mujeres, incluidas las amenazas de tales actos, la coacción o la privación arbitraria de la libertad, ya sea en la vida pública o en la vida privada. (p.2)

Esta definición fue ampliada en 1995 por la Cuarta Conferencia Mundial sobre la Plataforma de Acción de Beijing (Cruz y Klinger, 2011). Responde a varios factores sociales y culturales institucionalizados, complejos e interconectados, que han mantenido a las mujeres particularmente vulnerables a la violencia que les atañe, todas ellas manifestaciones de poder históricamente desiguales entre las relaciones entre hombres y mujeres (UNICEF, 2000). En consecuencia este tema ha recibido una creciente atención en las Naciones Unidas como una forma de discriminación y una violación de los derechos humanos de las mujeres; la comunidad internacional se ha comprometido a proteger los derechos y la dignidad de las mujeres y los hombres a título individual, mediante numerosos tratados y declaraciones. Las pruebas reunidas por los investigadores acerca de la difusión generalizada y las múltiples formas de violencia contra la mujer, unidas a campañas de promoción, desembocaron en el reconocimiento de que la violencia contra la mujer era de carácter mundial y sistémica y estaba arraigada en los desequilibrios de poder y la desigualdad estructural entre los hombres y las mujeres (Naciones Unidas, 2007).

Si la violencia contra las mujeres es la expresión más extrema de la desigualdad, parece difícil avanzar en su erradicación sin abordar la desigualdad estructural de género que atraviesa las sociedades latinoamericanas, y que se expresa en las limitaciones para el ejercicio de las autonomías física, económica y en la toma de decisiones; por eso, es importante preguntarse cómo se enmarcan las políticas sobre violencia contra las mujeres 
en relación con las políticas más amplias para promover la igualdad de género. Lo cierto es que son pocos los países de la región que han aprobado planes contra la violencia y que también cuentan con planes más amplios de igualdad de género, aunque éstos siempre incluyen como uno de sus componentes la lucha por la erradicación de la violencia (Gherardi, 2015).

La violencia doméstica es la forma más común de violencia contra la mujer. No existe una definición acordada internacionalmente de violencia doméstica que aborde el tema en su totalidad, pero las principales definiciones coinciden en que la violencia doméstica es violencia en la familia $o$ en la unidad doméstica, incluyendo, entre otras cosas, agresión física / maltrato; agresión / abuso mental, emocional y psicológico; violación y abuso sexual entre cónyuges, parejas regulares u ocasionales y cohabitantes (Unión Europea, 2010). Aunque las desigualdades de género son los principales mecanismos sociales detrás de la (re) producción de la violencia doméstica, las respuestas políticas a la violencia doméstica como un problema relacionado con el género varían tanto a nivel nacional como transnacional (Virkki, 2017).

Las políticas que abordan la violencia contra la mujer se expandieron dramáticamente en todo el mundo a finales de los 90 y la primera década de los años 2000 (Pierotti, 2013). En América Latina y el Caribe, hasta los años noventa, la violencia contra las mujeres, principalmente, la acaecida en el ámbito familiar, era considerada un asunto privado en el cual el Estado no debía intervenir; por otro lado, poco se conocía sobre la magnitud del problema, de manera que se tendía a asumir que la violencia hacia la población femenina ocurría de forma aislada, y no se la concebía como un problema social y de política pública (Camacho, 2014).

La multiplicidad de causas de la violencia contra la mujer (cultural, económica, legislativa, política y personal) requiere un enfoque político multidimensional que integre: la acción legislativa, la acción preventiva, la protección de las víctimas y los servicios de apoyo y reintegración que abarcan diferentes áreas de intervención; la población entera, así como el enjuiciamiento y el tratamiento de los perpetradores (Unión Europea, 2010).

Taus (2014) analiza los logros alcanzados en los últimos veinte años por el sistema interamericano de protección de los derechos humanos, a fin de dar solución al problema de la creciente, diversificada y multifacética violencia de género que impide la práctica — real y no meramente formal — de la igualdad y la no discriminación. Plantea que, desde un contexto regional y a partir de la vigencia del derecho internacional de los derechos humanos, el derecho inalienable a la igualdad es estudiado desde una perspectiva de género con el propósito de visualizar el ejercicio de jure y de facto del mismo.

\section{El contexto Ecuador}

Como lo señala Herrera (2001), luego de treinta años de haber sido acuñada como 
categoría de análisis social en la academia anglosajona, el género es todavía un campo de saber en construcción dentro de las Ciencias Sociales ecuatorianas; al igual que en muchos países latinoamericanos, en el Ecuador los estudios de género se caracterizan por una débil institucionalización en los centros académicos y una producción que proviene más bien de espacios extra académicos.

Ecuador cuenta con un marco legal y teórico favorable a nivel de derechos, lo cual puede generar una sensación de avance en la consecución de la equidad de género que, sin embargo, no se ajusta a los parámetros de realidad; dada la dificultad para desarrollar las leyes y hacer efectivas las garantías constitucionales. Es preciso observar que el reflejo de las inequidades de género en datos es complejo, pues más allá de la escasa actualización de datos en áreas de derechos sociales (salud, educación, trabajo, entre otras), y a pesar de los esfuerzos hechos gracias a la Ley de Transparencia y las estadísticas y consultorías realizadas por el Instituto Nacional de Estadísticas y Censos del Ecuador (INEC) en los últimos años, sigue existiendo una gran dificultad para incorporar la variable género en las encuestas (AACID, 2011).

Un referente importante para la discusión de la equidad de género en el Ecuador, lo constituye el diagnóstico realizado por Camacho (2014) para el Consejo Nacional para la Igualdad de Género. Este diagnóstico se basa en los resultados de la Encuesta Nacional sobre Relaciones Familiares y Violencia de Género contra las Mujeres, que fue realizada por primera vez en el Ecuador a finales del 2011; siendo este el segundo país de la región -después de México- en llevar a cabo una encuesta oficial sobre este problema social. El trabajo parte de un recuento de la respuesta estatal frente a la violencia hacia las mujeres y de la presentación del marco conceptual, normativo y metodológico. En el diagnóstico se concluye que, la violencia basada en el género constituye un problema social de gran magnitud, y que la violencia hacia las mujeres está presente en los distintos ámbitos del quehacer social (laboral, educativo, comunitario, de la salud, familiar), entre otros hallazgos.

Chávez y Juárez (2016) abordan el tema de violencia de género hacia la mujer perpetrado por su conviviente o ex conviviente, aplicando una metodología cuantitativa de diseño no experimental, tomando como sujetos de estudio treinta y siete mujeres de edades comprendidas entre los 20 a 55 años de edad, a quienes se aplicó el cuestionario de Respuesta Emocional a la Violencia Doméstica y Sexual, para conocer la respuesta emocional ante este problema. Los hallazgos reportados por estos autores, evidencian que las mujeres víctimas de violencia en general presentan afectación emocional, pero con niveles bajos de intensidad; y demuestra que en todos los tipos de maltrato hay presencia de agresión psicológica. Asimismo, los autores documentan que estas mujeres que han denunciado agresiones físicas permanecen viviendo con sus agresores porque asumen que es normal la agresividad, más el temor de quedarse solas, sus bajos niveles de educación, y sobre todo la carencia de 
ingresos que les imposibilita mantener a los hijos, mientras que hay otro grupo de mujeres que ya no conviven con sus agresores pero continúan siendo agredidas por sus ex convivientes.

Solís García et al. (2014) reportan una investigación donde presentan un enfoque sobre la perspectiva de género y analizan diversos problemas y situaciones relacionados con las diferentes construcciones sociales de lo femenino y lo masculino, las cuales se reflejan en lo social, en la sexualidad, en el trabajo remunerado, en el trabajo doméstico, en las políticas públicas y en la participación y organización social. Los autores concluyen que, en el estudio que realizaron, los directivos de las empresas son de sexo masculino, evidenciando una marcada desigualdad de género al negar las oportunidades a las mujeres para ocupar dichos cargos, y la carencia de políticas que promulguen la igualdad, a pesar del buen desempeño del sector femenino que se encuentra laborando.

\section{Reflexiones finales}

Tal como lo evidencia la revisión de literatura, el debate y diálogo público sobre estudios de género se han ampliado y profundizado en los últimos años, evidenciando, el logro de una mayor sensibilización y concientización social sobre los problemas que enfrentan las mujeres.

Los esfuerzos por consensuar una definición de igualdad de género, así como su legislación y soporte teórico por parte de instituciones como la UNESCO, Unión Europea, Naciones Unidas, y CEPAL, han permitido una consolidación de los estudios de género dentro de la comunidad científica. Esta amplia participación, ha hecho posible la visualización de los estudios de casos relacionados con la participación de la mujer en los espacios educativos, y la violencia de género en general.

Es hora de avanzar y protagonizar una nueva era, creando desde la familia, escuela, y la sociedad en el Ecuador, un nuevo código de equidad para la reconstrucción de una perspectiva de género basada en la igualdad de hombres y mujeres para erradicar las brechas psicológicas de la violencia, opresión e injusticia social que enfrentan las mujeres, por el bien de la humanidad para reaprender el concepto de libertad y justicia. Es indispensable profundizar más en el tema de equidad de género, para determinar los parámetros que inciden en el deterioro de las familias, en especial los índices de violencia y maltrato físico y psicológico de la mujer.

Aunque el desarrollo de estudios de género en Ecuador no ha sido prolífero, se espera que con el establecimiento de un marco legal y teórico, esta situación cambie en esta segunda década del siglo XXI. 


\section{Referencias}

Agencia Andaluza de Cooperación Internacional para el Desarrollo -AACID- (2011). Diagnóstico País Ecuador desde la perspectiva de Género. Recuperado de: http://www.juntadeandalucia.es/aacid/im ages/Servicios/Publicaciones/diagnostico _ecuador_genero.pdf

Aguiar, M.E., y Gutiérrez, H. (2017). Desigualdad de género y cambios sociodemográficos en México. Nóesis - Revista de Ciencias Sociales y Humanidades, 26 (51), 2-19.

Aikman, S., y Unterhalter, E. (2007). Practising Gender Equality in Education. Oxford: Oxfam GB. Recuperado de:

http://www.ungei.org/resources/files/Pro gIns_wholebook.pdf

Bobbitt, D. (2007).The gender income gap and the role of education. Sociology of Education, 80 (January), 1-22.

Buchmann, C., DiPrete, T, y McDaniel, A. (2008). Gender Inequalities in Education. Annual Review of Sociology, 34, 319-337.

Camacho, G. (2014). La violencia de género contra las mujeres en el Ecuador: Análisis de los resultados de la Encuesta Nacional sobre Relaciones Familiares y Violencia de Género contra las Mujeres. Consejo Nacional para la Igualdad de Género. Quito: Agencia Española de Cooperación Internacional para el Desarrollo -AECID-.

Castillo, M., y Gamboa, R. (2013). La vinculación de la educación y género. Revista Electrónica "Actualidades Investigativas en Educación”, 13 (1), 1-16.

Chávez, M., y Juárez, A. (2016). Violencia de género en Ecuador. Revista Publicando, 8, 104-115.

Contreras, F., Pedraza, J., y Mejía, X. (2012). La mujer y el liderazgo empresarial. Revista Diversitas - Perspectivas en Psicología, 8 (1), 183-194.
Comisión Europea (2016)

Strategicengagementforgenderequality 2016-2019. Bélgica: Unión Europea. Recuperado de: https://ec.europa.eu/anti-trafficking/sites/a ntitrafficking/files/strategic_engagement_f or_gender_equality_en.pdf

Comisión Económica para América Latina y el Caribe-CEPAL. (2016). Agenda $2030 y$ los Objetivos de Desarrollo Sostenible Una oportunidad para América Latina y el Caribe. Santiago: Naciones Unidas. Recuperado de http://repositorio.cepal.org/bitstream/han dle/11362/40155/15/S1700334_es.pdf

Cruz, A., y Klinger, S. (2011). Gender-based violence in the world of work: Overview and selected annotated bibliography. Working Paper 3. Geneva: International Labour Organization -ILO-. Recuperado de: http://www.ilo.org/wcmsp5/groups/public/--dgreports/---gender/documents/publication/ wcms_155763.pdf

Klasen, S., y Lamanna, F. (2009). The impact of gender inequality in education and employment on economic growth: new evidence for a panel of countries. Feminist Economics, 15, (3), 91-132.

Freidenvall, L. (2015). Gender equality in decision making: going beyond quotas, p. 55-58. En "Visions for Gender Equality" (Bettio F.,y Sansonetti, S., editors). Luxemburgo: Unión Europea. Recuperado de: https://www.researchgate.net/publication /282701993_Visions_for_Gender_Equali ty\#pf37.

García, E. (2000). Igualdad de género y desarrollo sostenible. FERMENTUN, 10 (27), 117-156.

Gherardi, N. (2015). Otras formas de violencia contra las mujeres que reconocer, nombrar y visibilizar. Serie: Asuntos de género. Santiago: Naciones Unidas. 
Herrera, G. (2001). Antología Género. Quito: FLACSO. Recuperado de:

http://www.flacso.org.ec/docs/antgenero. pdf.

Lombard, N. (2015). Gendered violence: a cause and a consequence of inequality, pp. 63-66. En "Visions for Gender Equality" (Bettio, F. y Sansonetti, S., editors).Luxemburgo: Unión Europea. Recuperado de:

https://www.researchgate.net/publication /282701993_Visions_for_Gender_Equali ty\#pf37.

Lorber, J. (2010). Gender Inequality - Feminist theories and politics. New York/Oxford: Oxford University Press.

Martínez, S., y Bivort, B. (2013).Los estereotipos en la comprensión de las desigualdades de género en educación, desde la psicología feminista. Psicologia\&Sociedade, 25, (3), 549-558.

Naciones Unidas (1993). Resolución de la Asamblea General: Declaración sobre la eliminación de la violencia contra la mujer. Nueva York. Consultado en:

http://www.acnur.org/fileadmin/scripts/doc. php?file=fileadmin/Documentos/BDL/200 2/1286

Naciones Unidas (2007). Poner fin a la violencia contra la mujer: De las palabras los hechos - Estudio del Secretario General. Nueva York: Naciones Unidas.

Naciones Unidas (2015). Objetivos de Desarrollo del Milenio, Informe de 2015, es hora de la acción mundial por las personas y el planeta. Nueva York: Naciones Unidas. Consultado en: http://www.un.org/es/millenniumgoals/p df/2015/mdg-report-2015_spanish.pdf

Ordorika, I. (2015). Equidad de género en la Educación Superior. Revista de la educación superior, 44 (174), 7-17.
Perrons, D. (2017). Gender and Inequality: Austerity and Alternatives. Intereconomics, 52 (1), 28-33.

Pierotti, R. (2013).Increasing rejection of domestic violence: Evidence of global cultural diffusion. American SociologicalReview, 78, 240-65.

Rodríguez, R., y Limas, M. (2017). El análisis de las diferencias salariales y discriminación por género por áreas profesionales en México, abordado desde un enfoque regional, 2015. Estudios Sociales, 27 (47), 120-150.

Shastri, A. (2014). Gender Inequality and Women Discrimination. Journal of Humanities and Social Science (IOSR-JHSS), 19, (11), 27-30.

Solís García, M., Rodríguez Angulo, D., Cárdenas Zea, M., Carranza Quimi, W., Quinaluisa Morán, V., Boza Valle,J., Cobo Litardo, E., Carreño Rodríguez, W., Murillo Campuzano, G., y Díaz Ocampo, E. (2014). Revista Científica y Tecnológica UPSE, 2, (1), 1-10.

Taus, P. (2014). La igualdad de género y el acceso a la justicia de las mujeres víctimas de violencia en la región dentro del sistema interamericano de protección de los derechos humanos. IUS, 8 (34), 21-41.

UNESCO (2015). La violencia de género relacionada con la escuela impide el logro de la educación de calidad para todos. Documento de política 17. París: UNESCO.

UNICEF (2000). Domestic violence against women and girls. Innocenti Digest, 6 . Florence, Italy: Innocenti Research Centre.

Unión Europea (2010). Violence against women and the role of gender equality, social inclusion and health strategies. Luxembourg: Publications Office of the European Union. 
Virkki, T. (2017). At the Interface of National and Transnational: The Development of Finnish Policies against Domestic Violence in Terms of Gender Equality. Social Sciences, 6, (31), 1-17.

World Health Organization. (2009).Promoting gender equality to prevent violence against women. Series of briefings on violence prevention: the evidence. Geneva: WHO Press. 